\section{Definición actual de la medicina interna y el internista}

\author{
LUZ M. LETELIER S. ${ }^{1}$, ANDRÉS VALDIVIESO D. ${ }^{2}$, RAIMUNDO GAZITÚA P. ${ }^{3}$, \\ SYLVIA ECHÁVARRI V. ${ }^{1,4,5}$, RODOLFO ARMAS M. ${ }^{6}$
}

\section{A consensus definition of internal medicine and the internist}

Internal Medicine is a basic clinical specialty in Medicine, but due to it's vast field of action it is quite difficult to define. This consensus article analyzes different definitions and proposes a current definition while analyzing several aspects of the specialty along with its strengths and weaknesses. We propose to define Internal Medicine as a clinical specialty devoted to the comprehensive care of adults, from adolescence to senility, particularly the diagnosis and non surgical treatment as well as primary and secondary prevention of their diseases, in hospital or ambulatory settings. We propose to define Internal Medicine as a clinical specialty devoted to the comprehensive care of adults in hospital or ambulatory settings, from adolescence to end of life, in terms of prevention, diagnosis and non-surgical treatments of disease.

(Rev Med Chile 2011; 139: 1081-1088).

Key words: Education, medical; Internal Medicine; Internist; Specialties, medical.

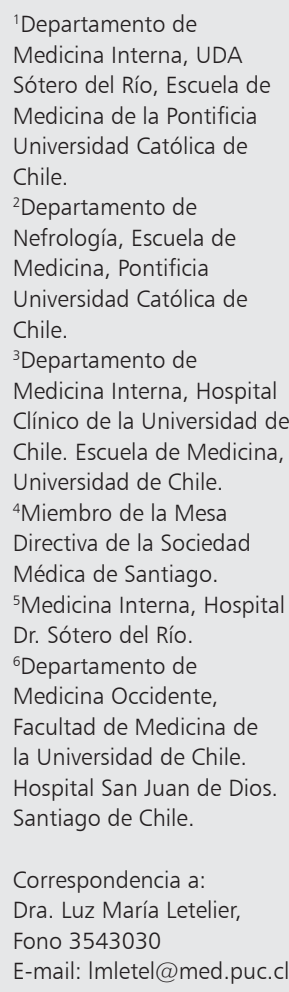

Documento redactado por el Grupo de Trabajo encargado para este efecto por la Mesa Directiva de la Sociedad Médica de Santiago-Sociedad Chilena de Medicina Interna (2007-2009). Aprobado como documento oficial por la Mesa Directiva de la Sociedad en junio de 2011.

\section{Soy internista ¿qué es eso?}

I as especialidades médicas surgen basadas en diferentes criterios: sistemas, edades, $\checkmark$ género, enfermedades o tecnologías ${ }^{1,2}$.

La Medicina Interna (MI), especialidad básica de la Medicina se refería a la disciplina que enfrentaba problemas de los órganos internos que el cirujano no podía abordar. Actualmente, cirujanos e internistas abordan patologías "externas" e "internas" y, aunque el nombre persiste, estaría obsoleto. El primer texto de medicina interna fue publicado por el médico alemán J. von Mering, en 1901 ("Lehrbuch der Inneren Medizin")

Como toda especialidad, cambia permanentemente; Sir W. Osler, uno de los padres de la medicina interna ${ }^{4}$, consideraba las necropsias parte de la MI. Una encuesta del American College of Physicians (ACP) a 990 internistas generales (IG) mostró que entre 1986 y 2004 disminuyó el número y variedad de procedimientos realizados por IG; procedimientos como paracentesis, tóracocentesis y punciones lumbares que realizaban la mayoría de los IG, el 2004 sólo las realizaban la cuarta parte de ellos ${ }^{5,6}$.

Su amplitud también genera confusión en la sociedad. Un ejemplo de esta confusión es el texto de Wikipedia ${ }^{7}$ que describe: "Debido al desprestigio de la especialidad en MI de múltiples orígenes, entre ellos el desconocimiento por parte de la sociedad, la advocación a tareas asistenciales rechazadas por otras especialidades por parte de las gerencias, y la desmotivación de muchos internistas, este es visto en muchos hospitales como el médico de los pacientes "desechados" por otras especialidades. Sin embargo, un servicio de MI, usado en toda su capacidad puede manejar el 90\% de la patología medica que llega al hospital y con el apoyo de los servicios centrales del hospital y un minimo grupo de superespecialistas hasta el 95\% de la patología médica de un hospital general. Son por ello los especialistas más versátiles, mejor formados y más eficientes en términos 
económicos de todas las especialidades. De ahí que en cualquier hospital por pequeño que sea, pueden faltar algunas especialidades pero siempre hay un servicio de M.I."

Es importante mirar periódicamente la MI, para adecuar los conocimientos y destrezas exigibles a estos especialistas ${ }^{8,9}$. La Directiva de la Sociedad Médica de Santiago-Sociedad Chilena de MI (SMS) consideró importante revisitar la MI y el rol del Internista del siglo XXI. Respondiendo a esta inquietud, intentaremos definir la MI y el internista actual, su ámbito de acción, sus principales características y diferencias con otras especialidades así como las fortalezas y debilidades de la especialidad. Para esto, convocamos un grupo representativo de internistas: IGs, becado de MI e internistas sub-especialistas (ISE). Buscamos definiciones de MI e internista en diversas fuentes y se analizaron para converger en una definición común.

\section{Definiciones}

Es difícil definir la MI, textos clásicos como el Harrison's ${ }^{10}$ no la define en cuanto a disciplina, si no que describe las características que debe tener, a nuestro juicio, cualquier médico. Nuestra búsqueda encontró variadas definiciones presentadas en las Tablas 1 y 2.

\section{¿Qué comparten y qué diferencias tienen las definiciones de MI? (Tabla 1)}

Especialidad o ciencia clínica: Todas consideran la MI como "especialidad médica" o "ciencia clínica" o "disciplina científica", es decir, no cualquier médico es especialista en MI, pues requiere preparación especifica.

Adultos: Término compartido por casi todas las definiciones, que por obvio no se define, pero ¿incluye los adolescentes? Los adolescentes podrían ser atendidos por pediatras, como en países donde los internistas atienden pacientes sobre 18 años. En Chile, el corte es a los 15 años, por lo cual serían manejados por pediatras e internistas sucesivamente; más aún, como muchos problemas del adolescente son biopsicosociales, también son vistos por médicos familiares (MF).

Otro aspecto debatible es el tipo de adulto a cargo de la MI, ¿el adulto sano y enfermo o sólo el enfermo? En el segundo escenario el foco principal sería el diagnóstico y tratamiento del adulto enfermo incluyendo prevención secundaria y terciaria, con poco énfasis en la prevención primaria que quedaría en manos de médicos generales y MF. Otros incluyen la prevención primaria en la definición de MI.

Patología no quirúrgica: Un enfermo no sabe si su enfermedad es "quirúrgica" o "médica", incluso a veces una primera evaluación médica (internista o cirujano) tampoco puede hacer esta diferencia. Muchas enfermedades tienen tratamientos médico-quirúrgicos (Ej. cánceres que requieren cirugía seguida de quimioterapia). Los avances médicos generan cambios terapéuticos, dejando el término "patologías quirúrgicas" obsoleto, (Ej. la úlcera péptica solía operarse, pero la endoscopia, los fármacos que inhiben la producción de ácido y el tratamiento del Helicobacter pylori, redujeron la indicación quirúrgica) o a la inversa, enfermedades que se consideraban de manejo médico tengan manejo quirúrgico. Así, parece más lógico el término "manejo no quirúrgico de las patologías", que "patologías no quirúrgicas". Esto condicionaría el campo de acción del Internista, por ejemplo, hospitales que tienen internistas a cargo del manejo médico de pacientes quirúrgicos.

Atención integral: Aspecto destacado de la MI, incluye la capacidad de diagnosticar y tratar patologías frecuentes y raras, así como patologías que afectan diversos órganos simultáneamente, reconociendo y derivando oportunamente cuando el paciente requiera tratamiento específico por otra especialidad (cirugía, psiquiatría, etc) o de una subespecialidad de la MI (p. ej: derivar al nefrólogo para ingreso a diálisis). Por cultivarse en ámbitos hospitalarios y ambulatorios de distinta complejidad la atención al paciente puede ser integral, independiente del número y gravedad de patologías.

Lugar de acción: Muchas definiciones lo omiten. Una considera la atención desde la atención primaria a la terciaria y otra considera sólo la atención de pacientes hospitalizados. Esta divergencia da cuenta de la amplitud del campo de acción de la MI así como de la confusión en su definición. En Chile, la atención de salud en el sistema público, 


\section{Tabla 1. Definiciones de medicina interna}

1. "Especialidad médica que se dedica a la atención integral del adulto enfermo, enfocada al diagnóstico y el tratamiento no quirúrgico de las enfermedades que afectan a sus órganos y sistemas internos, y a su prevención" ${ }^{3}$

2. "Especialidad médica que se ocupa de la atención integral del adulto enfermo, caracterizada por su forma de actuación clínica humanizada, no quirúrgica, que abarca desde la atención primaria a la atención terciaria"16

3.-"Ciencia clínica, central a las especialidades de la medicina y la cirugía que se ocupa de la salud y enfermedad en adolescentes y adultos" 17

4. Disciplina científica sobre el estudio, diagnóstico y tratamiento de enfermedades no quirúrgicas del paciente adolescente y adulto. Intrínseco a la disciplina son el profesionalismo y valores humanistas. Dominar la medicina interna requiere no sólo extenso conocimiento de fisiopatología, epidemiología e historia natural de la enfermedad si no también la adquisición de habilidades en entrevista medica, examen físico, relaciones humanas con pacientes y competencia en procedimientos $* 18$

5. Especialidad médica encargada del diagnóstico y tratamiento de enfermedades de los órganos internos del adulto*19

6. "Constituye el tronco del cual derivan todas las subespecialidades que enumeraremos a continuación. Sin embargo, es importante recordar que los internistas constituyen muy probablemente el primer encuentro del enfermo con la medicina. Todos nuestros médicos internistas subespecialistas, han desarrollado durante su formación un período de entrenamiento en Ml y están capacitados para actuar como médicos generales. Es así como los internistas pueden cubrir un amplio espectro de patologías que van desde las enfermedades más frecuentes que aquejan a nuestra población, hasta resolver delicados y complejos tratamientos"20

7. "La MI es una especialidad médica que se dedica a la atención integral del adulto enfermo, sobre todo a los problemas clínicos de la mayoría de los pacientes que se encuentran ingresados en un hospital. El médico que ejerce la especialidad "medicina interna" se llama médico internista"21

*traducida por los autores.

Tabla 2. Definiciones de internista

1. "Es el profesional médico especialista en MI (post-grado en un centro universitario y/o con certificación de la Comisión Nacional de Certificación de Especialidades Médicas), capacitado para promover la salud, prevenir y resolver los problemas derivados de las enfermedades del adulto. Es quien mejor posee las condiciones para formarse en una sub-especialidad clínica y en salud pública"16

2. Médicos especializados en la prevención, detección y tratamiento de enfermedades del adulto*22

3. "Dicho de un médico, que se dedica especialmente al estudio y tratamiento de enfermedades que afectan a los órganos internos" 23

4. "Internista en Chile, es el profesional que tras 7 años de estudio en la Facultad de Medicina sigue un Post-Grado de 3 años en un centro docente-asistencial con la certificación final de la Asociación de Facultades de Medicina de Chile (ASOFA$\mathrm{MECH}$ ). Se puede llegar a esta condición, tras el egreso de la Escuela de Medicina mediante un adiestramiento en práctica por un período de 5 años en un Servicio de Ml en un Hospital A, dando un examen de certificación ante la Comisión Nacional de Certificación de Especialidades Médicas (CONACEM). Este profesional al término de su formación debiera estar capacitado para tener las siguientes características: a. Conocimiento amplio de las enfermedades del adulto, desde la adolescencia a la senectud, con referencia a la etiología, clínica, prevención y tratamiento. b. Utilización criteriosa de los exámenes complementarios y procedimientos que facilitan el diagnóstico con sus indicaciones, rendimiento y riesgos. c. Manejo de la terapéutica farmacológica y de las indicaciones quirúrgicas con sus riesgos e interacciones. d. Mantención de la capacidad de estudio para renovar sus conocimientos en sistemas de educación continua. e. Desempeñarse en el Hospital en las salas de Medicina, Unidades de Cuidados Intensivos y Unidades de Emergencia, participando en reuniones clínicas. f. Desempeñarse en el control ambulatorio del paciente en el Consultorio Externo o en la consulta privada. g. Desempeñarse como docente idóneo en los cursos clínicos de Pregrado, con énfasis en la Semiología y en el Internado y el Post-grado de la especialización. h. Aplicar el método científico para realizar investigación clínica en su diario quehacer. i. Aplicar la Ética Médica que permite respetar los derechos de los pacientes y conocer las limitaciones de la terapéutica. j. Enfrentar el trabajo mediante la resolución por problemas y el conocimiento de la medicina basada en evidencias para resolver diagnósticos y tratamientos. k. Adquirir destreza en informática médica: búsqueda bibliográfica, bases de datos, estadísticas. I. Capacitarse en Gestión Administrativa para obtener el máximo rendimiento al menor costo, trabajando en equipos de profesionales médicos, paramédicos y otros profesionales"11

* traducida por los autores. 
se estratifica según su grado de complejidad en nivel primario, secundario y terciario. La atención terciaria está concentrada en hospitales de mayor complejidad, donde es claro el rol de la MI. Todo hospital terciario cuenta con Servicios de MI, que según la realidad local podría funcionar en 2 modalidades:

i. Servicios de MI indiferenciados atendidos por IG o ISE que mantengan las competencias del IG.

ii. Servicios de MI con camas asignadas a subespecialidades, atendidos por los correspondientes ISEs.

La primera es más coherente con la atención integral del paciente. La segunda, atrae por su aparente eficiencia, pero suele requerir de numerosas interconsultas, algunas innecesarias si el paciente fuere manejado por un IG.

El desarrollo de unidades de cuidados intermedios para pacientes de mediana gravedad, frecuentemente con polipatologías, ha generado un nuevo espacio laboral especialmente apropiado para el IG.

Algunos sugieren que los hospitales tengan servicios de adultos indiferenciados, independiente de la patología que motive la hospitalización. Estos pacientes serían vistos por internistas "hospitalistas" que evalúan al paciente integralmente y "dirigen la orquesta" respecto de la concurrencia de sub-especialidades médicas o quirúrgicas.
Más complejo es definir el rol de la MI en los niveles primarios y secundarios. Adultos con patología frecuente y poco compleja pueden ser manejados por IG, pero también podrían serlo por médicos generales y MFs como ocurre en nuestro sistema público de salud. Cuando estos pacientes tienen varias patologías o éstas se complican, serán derivados a la atención secundaria para ser manejados por IG o ISE según corresponda (Figura 1).

Central o tronco: Alude a la relación con las subespecialidades derivadas de MI.

Atención clínica humanizada: Característica de la MI, pero no privativo de ella.

Organos y sistemas internos: Este concepto parece inapropiado, aparte de los órganos de los sentidos, ¿cuales serian los sistemas "externos"?. La MI considera los distintos sistemas fisiológicos de forma integrada, los pacientes se analizan en base a problemas más que a diagnósticos aislados, obligando a considerar todos los sistemas del paciente.

\section{¿Que comparten y en que difieren} las definiciones de internista? (Tabla 2)

Las definiciones de internista del ACP y la Real Academia Española son tan amplias que varias disciplinas podrían ajustarse a ellas.

Concordamos con la definición de la SMS, en explicitar que es una especialidad clínica formal,

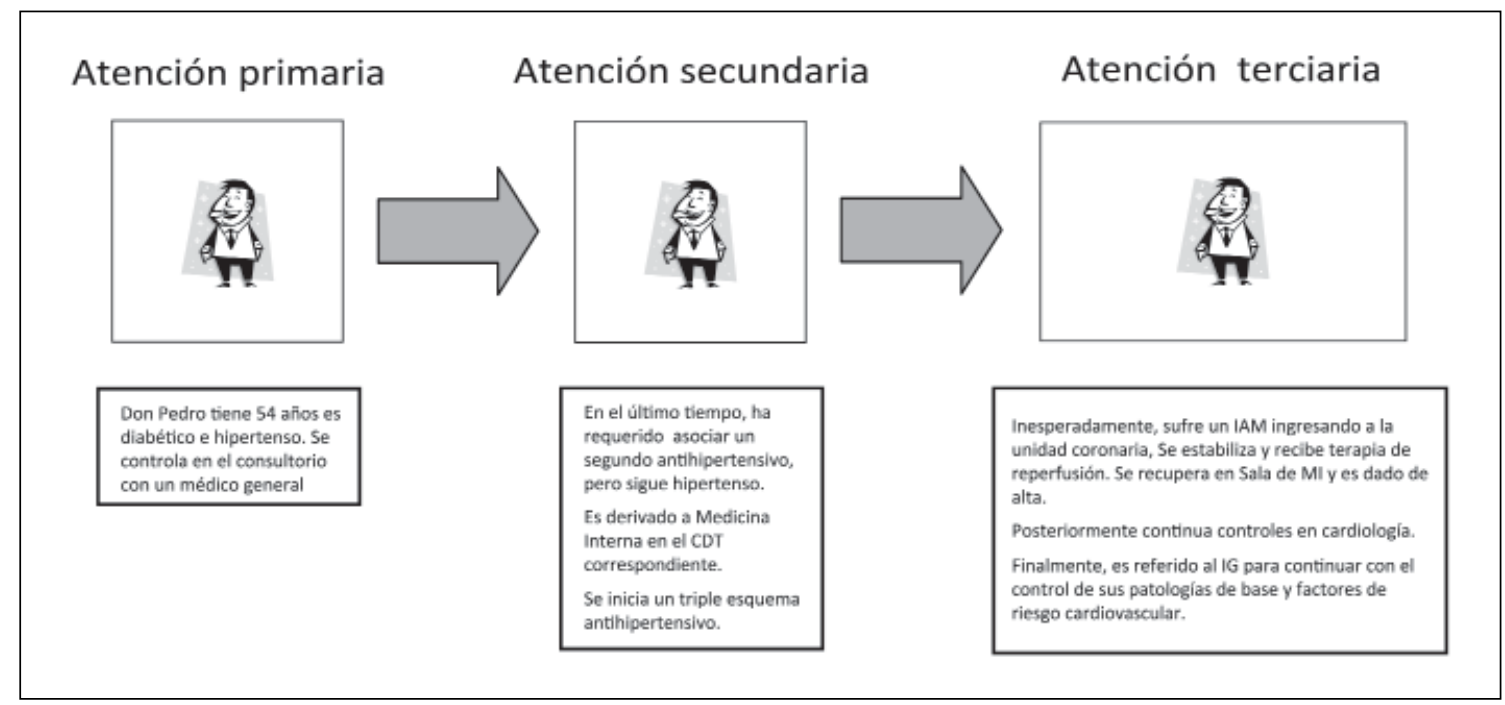

Figura 1. Modelo de atención de un paciente. 
pero requiere precisar algunos aspectos. El " $r e$ solver los problemas derivados de las enfermedades del adulto" deja el campo abierto a las patologías quirúrgicas. Considerando nuestro análisis de MI, el internista debería "diagnosticar las patologías del adulto y realizar el tratamiento no quirúrgico de éstas". Respecto de "es quien mejor posee las condiciones para formarse en una sub-especialidad clinica y en salud pública", debe precisarse que se refiere a sub-especialidades derivadas de la MI; pues el internista no está capacitado para otras especialidades clínicas (ej. anestesia cardiovascular). Respecto a salud pública, la MI es una de varias especialidades que pueden derivar a dicha especialidad, por lo que no correspondería a su definición.

La definición de Varela ${ }^{11}$ describe el proceso para ser considerado internista en Chile y enumera las características que debe poseer. A nuestro juicio, algunas corresponden a todo médico:

1. Aplicar principios bioéticos.

2. Profesionalismo.

3. Comunicación empática.

4. Capacidad de actualizar conocimientos.

5. Aplicación de medicina basada en evidencia.

6. Habilidades docentes.

7. Liderazgo.

\section{Competencias más propias del internista}

1. Experto en enfermedades del adulto, considerando su etiología, enfrentamiento clínico (semiología y diagnóstico) prevención y tratamiento. Incluyendo algunas patologías neurológicas, psiquiátricas y ginecológicas de diagnóstico y tratamiento menos complejo.

2. Capacidad de manejo integral de los pacientes con enfoque global que abarque cada sistema fisiológico y aspectos psicosociales.

3. Desempeñarse en ambientes ambulatorios y hospitalarios.

\section{Definiciones propuestas por los autores y respaldadas por la Directiva de la Sociedad Médica de Santiago}

\section{Medicina Interna}

Especialidad clínica dedicada a la atención integral del adulto, desde la adolescencia hasta la senectud, con énfasis en el diagnóstico y tratamiento no quirúrgico de sus patologías y en la prevención primaria y secundaria de éstas, tanto en ambiente hospitalario como ambulatorio.

\section{Internista}

Médico capacitado para prevenir, diagnosticar y aplicar el tratamiento no quirúrgico de las patologías del adulto. Puede desempeñarse desde la atención ambulatoria hasta un intensivo; atendiendo a enfermos con una o más patologías crónicas o agudas. Tiene las condiciones necesarias para formarse en una subespecialidad derivada de MI.

\section{Disciplinas con que se confunde}

Es común que la MI se superponga y confunda con otras ramas de la medicina, por su amplio campo de acción, estar estrechamente ligada con sus sub-especialidades y muy representada en el currículo del pregrado médico. La sociedad reconoce fácilmente al pediatra, cirujano o ginecólogo, pero confunden al médico general, médico familiar, el IG y el ISE.

\section{Médico general vs IG}

Confusión frecuente por la similitud de los términos, aunque su rol y formación son esencialmente diferentes. En Chile, se denomina médico general a quien egresó con el título de médico cirujano, este profesional es más bien un médico indiferenciado con potencial para alcanzar mayor desarrollo, en cambio, IG es un médico que realizó 3 años de especialización. El médico general atiende adultos y niños con patología prevalente, generalmente simple y de manejo ambulatorio, propia de establecimientos asistenciales de baja complejidad, a diferencia del IG que maneja sólo adultos, con patologías simples o complejas y en ambiente ambulatorio y hospitalario.

\section{MF vs IG}

Ambas son especialidades clínicas de 3 años, después del titulo profesional. El foco del MF, que considera la atención de adultos y niños, está en la prevención primaria y manejo del grupo familiar en un ambiente ambulatorio. El IG no atiende niños y su foco principal esta más en el diagnóstico y tratamiento de las patologías del adulto, tanto en ambientes ambulatorios como hospitalarios. Inevitablemente comparten algunas áreas, particularmente con la MI ambulatoria. 


\section{IG vs ISE}

En Chile y otros países, luego de formarse en MI se puede optar al entrenamiento por dos años en una sub-especialidad derivada de la MI. Estas podrían clasificarse en "verticales", aquellas referidas a un sistema como, Cardiología o Nefrología y "horizontales" las referidas a una situación particular como Geriatría o Medicina Intensiva.

$\mathrm{Si}$ estos subespecialistas se han formado primero como internistas, la diferenciación sólo tiene sentido entre el IG y el ISE. No hay consenso en la denominación de los internistas que optan por no hacer subespecialidad y se dedican a la MI en su conjunto. La expresión "médicos generalistas" se confunde con los médicos generales, "internistas indiferenciados" produce la sensación de que no seria una especialidad. Los llamaremos Internistas Generales.

Distinguir el rol del IG respecto del ISE es importante. El IG diagnosticará la mayoría de las patologías del adulto, tratará aquellas de mayor prevalencia y solicitará una interconsulta o derivará al paciente oportunamente cuando el tipo o etapa de la enfermedad requieran manejo especializado. Ciertamente, la visión amplia del IG otorga ventajas comparativas en el aspecto diagnóstico respecto al ISE. El ISE comparte las características generales del internista, pero profundiza el conocimiento en un área específica de la MI desarrollando competencias para resolver patologías con técnicas diagnósticas o terapéuticas propias de esa sub-especialidad. La subespecialización llega a niveles sorprendentes ${ }^{2}$, como internista, cardiólogo y arritmólogo, diestro en electrofulgurar determinadas arritmias.

Los pacientes con polipatologías y múltiples terapias, requieren varios ISE simultáneamente, pudiendo llevar a una incorrecta jerarquización de patologías o a indicaciones terapéuticas con interacciones riesgosas. En dichos casos el IG cumple un rol fundamental: mantiene una visión global e integrada de las patologías del paciente, actuando como "médico de cabecera" e interactuando con el enfermo, su familia y los ISE.

\section{$¿$ Debe un sub-especialista formarse primero en MI?}

Sería atractivo graduar un sub-especialista de MI en 3 años, sin pasar por el rigor, y esfuerzo de la formación previa en MI, como ocurre en España por ejemplo. A nuestro juicio la formación en
MI tiene ventajas tan importantes, que la hacen indispensable.

a. Dado que la MI existe y tiene un "contenido real", sería conceptualmente previa y superior a las subespecialidades. Estas en cierto modo "le pertenecen". Las partes no pueden ser superiores al todo: el conjunto aporta herramientas indispensables para conocer las partes.

b. El entrenamiento en MI, puede iniciarse antes de haber decidido una subespecialidad, constituyendo un tiempo de maduración para evaluar opciones, incluyendo ser IG.

c. El entrenamiento en MI crea buenos "hábitos" semiológicos. La sistematización del enfrentamiento clínico es fundamental para cualquier clínico. Una buena historia, un completo pero eficiente examen físico, lo entrena mejor la MI que la subespecialidad. El pregrado, no expone al alumno lo suficiente para adquirir indeleblemente dichas destrezas.

d. El enfrentamiento clínico, uno de los desafíos del IG, coincide con el proceso que realizará el ISE. Sin embargo, las enfermedades suelen afectar varios sistemas, requiriéndose un proceso mental integrador y jerarquizado. Un subespecialista sin entrenamiento en MI, pierde dicha perspectiva, olvidando que algunos síntomas pueden originarse en "partes" alejadas de su campo, pudiendo solicitar exámenes innecesarios, cuyo rendimiento podría desconocer.

e. Los tratamientos frecuentemente afectan otros sistemas. Es necesario conocer los riesgos de la polifarmacia a la que sometemos a los pacientes. Simplificar un tratamiento, lo enseña mejor la MI que la subespecialidad.

f. La preparación en d y e reducirían los riesgos del paciente y los costos en salud.

g. Un subespecialista formado en MI tendrá conocimientos más amplios que los de su campo, con "más cultura médica" y mayor flexibilidad para los cambios de la medicina del futuro.

En suma, un subespecialista con formación en MI, tiene mejor entrenamiento y más oportunidad de comprender globalmente al enfermo.

\section{Fortalezas y debilidades del IG}

\section{Fortalezas}

1. Manejar integralmente al adulto enfermo con distintas patologías simultáneamente. 
2. Acompañar al paciente en distintas etapas de su vida y de su enfermedad: enfermedades simples de manejo ambulatorio o graves manejadas en servicios de alta complejidad.

Estas fortalezas son particularmente importantes porque el aumento en la expectativa de vida genera frecuentemente polipatologías crónicas. Del punto de vista del paciente y del sistema de salud, es fundamental una especialidad que se encargue del paciente en su conjunto. El IG sería el añorado "médico de cabecera", a cargo del enfermo más que de sus enfermedades. El ISE complementaría el manejo integral del adulto enfermo.

Otra consideración es el impacto de las patologías crónicas en los distintos órganos. Clásico ejemplo es la diabetes; el diabetólogo trata al diabético, pero cuando éste tiene compromiso cardiaco o renal pasa a ser manejado por cardiólogo y nefrólogo, requiriendo de 3 tratantes; esto complica al enfermo y encarece los costos en salud. Los ISE pueden mantener las fortalezas de los IG, pero generalmente la super subespecialización dificulta mantenerlas; tampoco está resuelto cual sería la mejor forma de lograrlo, reforzando el rol del IG.

\section{Debilidades}

Competencia entre IG e ISE

1. Las subespecialidades fueron constituyéndose en amenazas al $\mathrm{IG}^{12,13}$ al ser preferidas por internistas que consideraban más fácil y eficiente conocer profundamente una información restringida, favoreciendo la sobre-representación de ISE. Es difícil determinar cuando termina el accionar del IG y comienza el ISE. Es claro que el IG debe ser capaz de diagnosticar la mayoría de las patologías del adulto, pero no es menos cierto que el IG requiere a veces tempranamente del concurso terapéutico del ISE.

2. Algunas sub-especialidades incluyen técnicas (hemodinámicas, endoscópicas, dialíticas, etc.), que además de atractivas en sí mismas, representan mayores ingresos económicos. Lamentablemente, a veces hacen focalizarse en una determinada patología/técnica dificultando el enfoque integral como internista.

3. El desarrollo de ISE también se sustenta en que la población siente más seguridad si su médico se dedica especialmente a lo que le afecta. No obstante, los pacientes reconocen la dificultad de tener varios médicos simultáneamente.
Ingresos y reconocimiento

En general la renta del internista es menor que otras especialidades médicas, particularmente aquellas con procedimientos quirúrgicos. $\mathrm{Al}$ interior de la MI también hay diferencias entre los ingresos del IG y el ISE, particularmente si realizan procedimientos ${ }^{14}$.

\section{Interés en la especialidad}

El rigor y amplitud de la MI, genera la sensación de ser "inabarcable". Como consecuencia, en EEUU, el interés entre los egresados de medicina ha declinado notoriamente, especialmente en el ejercicio ambulatorio de la MI general ${ }^{15}$. En Chile, se mantiene el interés por la MI entre los egresados, sin embargo, al finalizar sus 3 años de MI muchos optan por una subespecialidad. Posiblemente esto refleja una brecha entre los programas de formación y la realidad de su ejercicio. En efecto, la enseñanza desmedidamente intrahospitalaria, basada en pacientes muy graves, contrasta con el ejercicio de una MI que comúnmente es ambulatoria y con pacientes crónicos deseosos que su médico asuma la atención integral que necesita. Incluso a veces la formación de internistas queda en manos de ISE en vez de IG llevando a los jóvenes a repetir el modelo. En nuestra experiencia, esta tendencia cambia en la medida que los residentes de MI son expuestos al modelo de trabajo de IG.

\section{Conclusiones}

La definición de MI e Internista cambian según las necesidades de la población y el desarrollo de la medicina, haciendo necesario revisar periódicamente estos conceptos. Las definiciones propuestas en este documento se acercan a la realidad actual de esta especialidad.

\section{Referencias}

1. Espinoza BA. La Medicina Interna y los internistas en la búsqueda "resiliente" de su identidad. Revista Electrónica de las Ciencias Médicas en Cienfuegos, MEDISUR 2008; 6: 2-9.

2. Cassel CK, Reuben DB. Specialization, subspecialization, and sub-subspecialization in internal medicine. $\mathrm{N}$ Engl J Med 2011; 364: 1169-73.

3. Reyes H. ¿Que es Medicina Interna? Rev Med Chile 2006; 134: $1338-44$. 
4. Osler W. The Principles and Practice of Medicine. D Appleton and Co New York, 1892.

5. Wigton Rs, Alguire P. The declining number and variety of pro-cedures done by general internists: a resurvey of members of the American College of Physicians. Ann In-tern Med 2007; 146: 355-60.

6. Duffy FD, Holmboe ES. What procedures should internists do? Ann Intern Med 2007; 146: 392-3.

7. Wikipedia. Lo que es y no es la medicina interna. http:// es.wikipedia.org/wiki/Medicina_interna (consultado febrero 2011).

8. Valdivieso DV. El internista, hoy. Rev Med Chile 1995; 123: 641-5.

9. Goic A. Formación del Médico Internista. Rev Med Chile 1996; 124: 740-6.

10. "Chapter 1. The Practice of Medicine" (Chapter). Fauci AS, Braunwald E, Kasper DL, Hauser SL, Longo DL, Jameson JL, Loscalzo J: Harrison's Principles of Internal Medicine, 17e: disponible en http://www.accessmedicine.com/content.aspx?aID $=2858000$ (consultado 13 marzo, 2011).

11. Varela N. Ejercicio de la Medicina Interna en América Latina. Rol del Médico Internista. Rev Med Chile 2002; 130: 96-100.

12. Duclós J. ¿Profesión? Médico. ¿Especialidad? Internista. ¿Nada más?. Nada más. Rev Med Chile 1999; 127: 481-4.

13. Sabah D. La crisis de la Medicina Interna. Rev Méd Chile 1996; 124: 1524-7.

14. Horn L, Tzanetos K, Thorpe K, Straus SE. Factors associated with the sub-specialty choices of internal medicine residents in Canada. BMC Med Educ 2008;
26; 8: 37. Disponible en http://www.biomedcentral. com/1472-6920/8/37 (consultado 10 marzo 2011).

15. Association of Program Directors in Internal Medicine, Fitzgibbons JP, Bordley DR, Berkowitz LR, Miller BW, Henderson MC. Redesigning residency education in internal medicine: a position paper from the Association of Program Directors in Internal Medicine. Ann Intern Med 2006; 144: 920-6.

16. Definiciones de Medicina Interna, Internista, Misión y Visión de la Sociedad Médica de Santiago (2006) pagina web Sociedad Médica de Santiago, disponible en http:// www.smschile.cl/index.php?id=155 (consultado 12 de marzo, 2011).

17. Gómez J. La Medicina Interna: situación actual y nuevos horizontes. Anales de Medicina Interna 2004; 21: 301-5.

18. Kelley's textbook of Internal Medicine. Chapter 1: Introduction to Internal Medicine as a discipline, pg 2.

19. National Library of Medicine disponible en http://www. ncbi.nlm.nih.gov/meshterm=internal\%20medicine (consultado 12 de marzo, 2011).

20. Pagina web Clínica Las Condes, disponible en http:// www.clinicalascondes.cl/Especialidades/MedicinaInterna. (consultado 10 de marzo, 2011).

21. Wikipedia, Medicina Interna. Disponible en http:// es.wikipedia.org/wiki/Medicina_interna (consutado 10 de marzo, 2011).

22. The American College of Physicians disponible en http://www.acponline.org/about_acp/ (consultado 10 de marzo, 2011).

23. Real Academia Española disponible en http://buscon.rae. es/ (consultado 10 de marzo, 2011). 